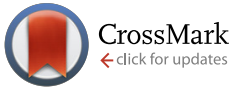

Cite this: Med. Chem. Commun., 2015, 6, 1471

\title{
Transition metal diamine complexes with antimicrobial activity against Staphylococcus aureus and methicillin-resistant $S$. aureus (MRSA) $\dagger$
}

\author{
G. W. Karpin, ${ }^{a b}$ D. M. Morris, ${ }^{a b}$ M. T. Ngo, ${ }^{a b}$ J. S. Merola*ab and J. O. Falkinham $\|^{a c}$
}

\begin{abstract}
Pentaalkylcyclopentadienyl $\left(C \mathrm{p}^{\mathrm{R}}\right)$ iridium (Ir) and cobalt (Co) 1,2-diamine complexes were synthesized. Susceptibility of Staphylococcus aureus and recent patient methicillin-resistant S. aureus (MRSA) isolates to the transition metal-diamine complexes were measured by broth microdilution and reported as the MIC and MBC. Hemolytic activities of the transition metal-complexes as well as toxicity toward Vero cells were also measured. The transition metal complex of $\mathrm{Cp}^{* \mathrm{R}} \mathrm{Ir}$ with cis-1,2-diaminocyclohexane, had strong antibiotic activity against $S$. aureus and MRSA (MIC $=4 \mu \mathrm{g} \mathrm{mL}^{-1}, \mathrm{MBC}=8 \mu \mathrm{g} \mathrm{mL}^{-1}$ ) strains and killed $99 \%$ of $S$. aureus cells in 6 hours. Stronger antibiotic activity was associated with the presence of octyl linked to the cyclopentadienyl group and cyclohexane as the diamine backbone. Activity was greatly diminished by trior tetramethylation of the nitrogen of the diamine. A cyclopentadienylcobalt complex of cis-1,2-diaminocyclohexane also showed significant anti-microbial activity against both $S$. aureus and MRSA strains. The absence of hemolytic activity, Vero cell cytotoxicity and the significant anti-microbial activity of several members of the family of compounds reported suggest this is an area worth further development.
\end{abstract}

Received 27th May 2015,
Accepted 18th June 2015
DOI: 10.1039/c5md00228a
www.rsc.org/medchemcomm and co-workers have synthesized and investigated the cytotoxic properties of a number of half-sandwich type organometallic compounds. ${ }^{9,10}$ Others have also reported on the cytotoxic behavior of different variants of half-sandwich compounds. ${ }^{11,12}$

By comparison, the work on small inorganic/organometallic compounds as antimicrobials has been sparse. ${ }^{13}$ The antimicrobial properties of the $\mathrm{Ag}^{+}$ion have long been known. ${ }^{14,15}$ More recently new compounds incorporating the silver ion with ligands such as $N$-heterocyclic carbenes have been shown to possess anti-microbial activity as well. ${ }^{16-18}$ In many other cases, anti-bacterial activity stems from either making a metal complex of a known organic anti-bacterial $^{19,20}$ or making an organometallic analog of a known antibiotic by replacing an aryl group with an organometallic moiety such as ferrocene. ${ }^{21,22}$ Recently, an intriguing polypeptide with three organometallic groups has been shown to be a potent antimicrobial. ${ }^{23} \mathrm{~A}$ review on small organometallic compounds as antibacterial agents appeared recently. ${ }^{24}$

Previously, we have reported that compounds based on simple organometallic complexes comprised of either iridium (Ir) or rhodium (Rh) chelated by amino acids were potent in vitro inhibitors of members of the genus Mycobacterium. ${ }^{25}$ Herein we extend our documentation of the strong antibiotic properties of a different family of transition metal complexes containing 1,2-diamine ligands to $S$. aureus and MRSA. Based on the encouraging initial results we believe that this work could lead to the identification of new therapeutic targets 
and development of a new class of urgently needed drugs to address the growing problem of difficult-to-treat $S$. aureus and MRSA infections.

\section{Results and discussion}

\section{Chemistry}

In a previous paper, we reported on cyclopentadienyl rhodium and iridium amino acid complexes that exhibited significant anti-mycobacterial activity. ${ }^{25}$ None of the amino acid compounds displayed any significant anti-staphylococcal activity. As part of that mycobacterial study, we also made complexes of two well-known drugs used in the treatment of tuberculosis (TB): isoniazid and ethambutol. ${ }^{26}$ Neither $\left[\mathrm{Cp}^{*} \operatorname{Ir}(\right.$ isoniazid $\left.) \mathrm{Cl}\right] \mathrm{Cl}$ nor $\left[\mathrm{Cp}^{*} \operatorname{Ir}(\right.$ ethambutol$\left.) \mathrm{Cl}\right] \mathrm{Cl}$ were active against an extensive panel of mycobacteria with MIC > $125 \mu \mathrm{g} \mathrm{mL}{ }^{-1}$. However, our practice is to screen all compounds first against a broad panel of bacteria, yeast and fungi and we noted that the ethambutol compound had moderate activity against $S$. aureus (MIC $=35 \mu \mathrm{g} \mathrm{mL}{ }^{-1}$ ). Noting that ethambutol is a substituted 1,2-diaminoethane, we turned our attention to complexes of other diamine ligands. That replacement of the chelating amino acids with chelating 1,2-diamines was successful in producing the antistaphylococcal compounds described in this work.

Complexes of rhodium and iridium were synthesized according to the general procedure outlined in Scheme 1. The penta-alkyl substituted $\mathrm{Cp}$ rings shown in the scheme may be pentamethyl $\left(\mathrm{Cp}^{*}\right)$ or tetramethylalkyl $\left(\mathrm{Cp}^{* \mathrm{R}}\right)$ the starting cyclopentadienes being synthesized as described previously. ${ }^{27}$ Using this route, an extensive series of compounds were prepared, fourteen of which are listed in Table 1, 1-Ir through 14-Ir. No significant difference in activity was found between $\mathrm{Rh}$ and Ir compounds, so only Ir compounds are reported here.

It was also decided that the cobalt congeners should be investigated since the lighter, first-row transition elements would be less likely to have general cytotoxic behaviors. The route shown in Scheme 1 is not applicable for cobalt, so a

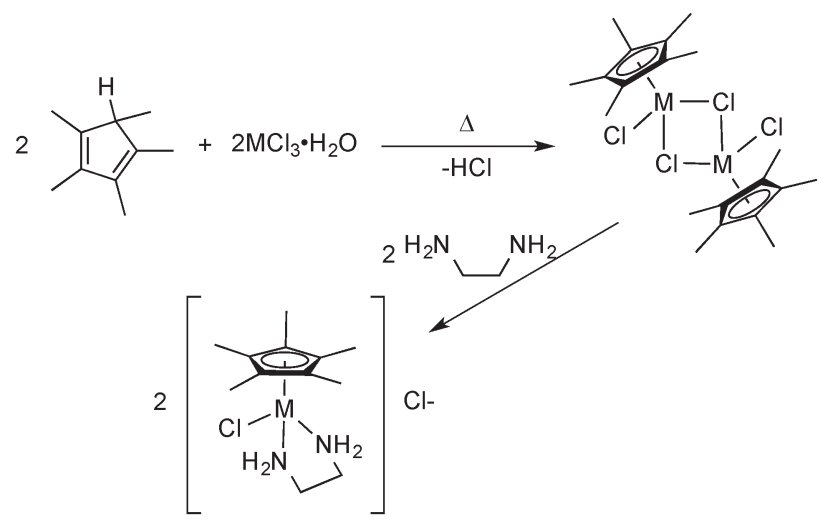

Scheme 1 Outline of the 2-step synthesis of iridium complexes with 1,2-diamines as the organic ligand. Ring and diamine substitution is delineated in Table 1.
Table 1 General structure and numbering for complexes in this paper. The table lists the various diamine ligand substitutions $\left(R_{1}, R_{2}, R_{3}\right.$ and $\left.R_{4}\right)$, the $\mathrm{C}_{5} \mathrm{Me}_{4} \mathrm{R}$ substitution as well as the metal

\begin{tabular}{|c|c|c|c|c|c|c|}
\hline & & & $\begin{array}{r}\mathrm{R}_{5} \\
\mathrm{R}_{5} \\
\mathrm{X}- \\
\mathrm{R}_{4}- \\
-\quad \mathrm{R}_{3}\end{array}$ & $\left.\begin{array}{l}-R_{6} \\
R_{5} \\
R_{2} \\
-R_{1}\end{array}\right] x$ & & \\
\hline Cpd & $\begin{array}{l}\mathrm{R}_{1}, \mathrm{R}_{2} \\
\mathrm{R}_{3}\end{array}$ & $\mathrm{R}_{4}$ & $\mathrm{R}_{5}$ & $\mathrm{R}_{6}$ & $\mathrm{X}$ & Ligand \\
\hline 1-Ir & $\mathrm{H}$ & $\mathrm{H}$ & $\mathrm{CH}_{3}$ & $\mathrm{CH}_{3}$ & $\mathrm{Cl}$ & $-\mathrm{CH}_{2}-\mathrm{CH}_{2}^{-}$ \\
\hline 2-Ir & $\mathrm{H}$ & $\mathrm{H}$ & $\mathrm{CH}_{3}$ & $\mathrm{C}_{8} \mathrm{H}_{17}$ & $\mathrm{Cl}$ & $-\mathrm{CH}_{2}-\mathrm{CH}_{2}-$ \\
\hline 3-Ir & $\mathrm{H}$ & $\mathrm{H}$ & $\mathrm{CH}_{3}$ & $\mathrm{CH}_{3}$ & $\mathrm{Cl}$ & cis-Cyclohexane \\
\hline 4-Ir & $\mathrm{H}$ & $\mathrm{H}$ & $\mathrm{CH}_{3}$ & $\mathrm{C}_{8} \mathrm{H}_{17}$ & $\mathrm{Cl}$ & cis-Cyclohexane \\
\hline 5-Ir & $\mathrm{H}$ & $\mathrm{H}$ & $\mathrm{CH}_{3}$ & $\mathrm{C}_{6} \mathrm{H}_{13}$ & $\mathrm{Cl}$ & cis-Cyclohexane \\
\hline 6-Ir & $\mathrm{H}$ & $\mathrm{H}$ & $\mathrm{CH}_{3}$ & $\mathrm{CH}_{3}$ & $\mathrm{Cl}$ & trans-Cyclohexane \\
\hline 7-Ir & $\mathrm{H}$ & $\mathrm{H}$ & $\mathrm{CH}_{3}$ & $\mathrm{C}_{8} \mathrm{H}_{17}$ & $\mathrm{Cl}$ & trans-Cyclohexane \\
\hline 8-Ir & $\mathrm{H}$ & $\mathrm{H}$ & $\mathrm{CH}_{3}$ & $\mathrm{C}_{12} \mathrm{H}_{25}$ & $\mathrm{Cl}$ & cis-Cyclohexane \\
\hline 9-Ir & $\mathrm{CH}_{3}$ & $\mathrm{CH}_{3}$ & $\mathrm{CH}_{3}$ & $\mathrm{CH}_{3}$ & $\mathrm{Cl}$ & $-\mathrm{CH}_{2}-\mathrm{CH}_{2}^{-}$ \\
\hline 10-Ir & $\mathrm{CH}_{3}$ & $\mathrm{H}$ & $\mathrm{CH}_{3}$ & $\mathrm{CH}_{3}$ & $\mathrm{Cl}$ & $-\mathrm{CH}_{2}-\mathrm{CH}_{2}^{-}$ \\
\hline 11-Ir & $\mathrm{H}$ & $\mathrm{C}_{7} \mathrm{H}_{7}$ & $\mathrm{CH}_{3}$ & $\mathrm{CH}_{3}$ & $\mathrm{Cl}$ & $-\mathrm{CH}_{2}-\mathrm{CH}_{2}^{-}$ \\
\hline 12-Ir & $\mathrm{H}$ & $\mathrm{C}_{10} \mathrm{H}_{7}$ & $\mathrm{CH}_{3}$ & $\mathrm{C}_{8} \mathrm{H}_{17}$ & $\mathrm{Cl}$ & $-\mathrm{CH}_{2}-\mathrm{CH}_{2}^{-}$ \\
\hline 13-Ir & $\mathrm{H}$ & $\mathrm{H}$ & $\mathrm{CH}_{3}$ & $\mathrm{CH}_{3}$ & $\mathrm{Cl}$ & $\begin{array}{l}(1 S, 2 S)-1,2-B i s(4- \\
\text { fluorophenyl) }\end{array}$ \\
\hline 14-Ir & $\mathrm{H}$ & $\mathrm{H}$ & $\mathrm{CH}_{3}$ & $\mathrm{CH}_{3}$ & $\mathrm{Cl}$ & $\begin{array}{l}(1 S, 2 S)-1,2-\operatorname{Bis}(4- \\
\text { methoxyphenyl) }\end{array}$ \\
\hline 15-Co & $\mathrm{H}$ & $\mathrm{H}$ & $\mathrm{H}$ & $\mathrm{H}$ & I & cis-Cyclohexane \\
\hline 16-Co & $\mathrm{H}$ & $\mathrm{H}$ & $\mathrm{H}$ & $\mathrm{H}$ & I & trans-Cyclohexane \\
\hline
\end{tabular}

new synthesis based on cyclopentadienylcobaltdicarbonyl, $\mathrm{CpCo}(\mathrm{CO})_{2}$, was developed ${ }^{28}$ (Scheme 2). Only the 1,2diaminocyclohexane compounds of cobalt, 15-Co and 16-Co, are discussed herein.

\section{Characterization of compound structure and purity}

All compounds reported in this paper were characterized by standard analytical techniques: ${ }^{1} \mathrm{H}$ NMR spectroscopy, ${ }^{13} \mathrm{C}$ NMR spectroscopy and High Resolution Mass Spectrometry. All spectroscopies were completely consistent with the proposed structures. In addition, to assure the purity of the complexes by examining the NMR spectroscopies for the presence of any contaminants, such as solvents, compounds were submitted for $\mathrm{C}, \mathrm{H}$ analyses which showed purity within $0.4 \%$ of calculated values.

All complexes described in this paper are quite water soluble, even complex 12-Ir containing a decyl chain on the Cp ligand. This is not surprising since the compounds are cationic with a chloride counterion. Complexes that had been

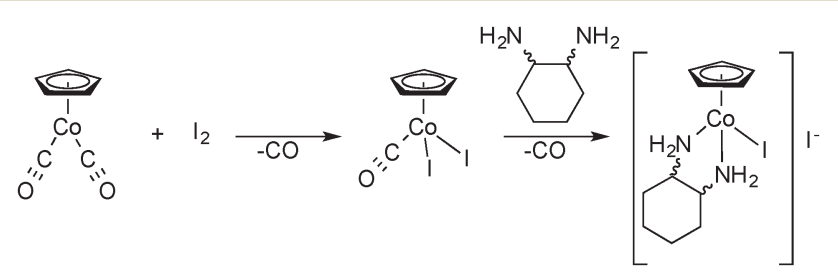

Scheme 2 Outline of the synthesis of cobalt-containing complexes with 1,2-diaminocyclohexane. 
dissolved in $\mathrm{D}_{2} \mathrm{O}$ were monitored by ${ }^{1} \mathrm{H}$ NMR spectroscopy and show no changes in physical appearance of the solution nor any change in the NMR spectra over months of monitoring. An indicator that, in general, these diamine complexes are stable in these in vitro conditions is that there was no anti-mycobacterial activity of the ethambutol complex. At the highest concentrations used, even a few percent decomposition of the ethambutol complex would release a sufficient amount of free ethambutol to show anti-mycobacterial activity and none was observed.

More to the point, a standard solution of complex 5-Ir in the growth medium was prepared and tested for activity against $S$. aureus at the time of preparation, at 6 months following preparation and at one year following preparation. The standard solution was maintained at ambient temperature during that period of time. The MIC of 5-Ir against $S$. aureus was reproducibly $4 \mu \mathrm{g} \mathrm{mL}^{-1}$ over the one year testing period showing no decomposition of the complex in the culture medium over a 12 month period of time.

\section{Anti-staphylococcal activities and complex structures}

Sixteen different complexes that were tested against $S$. aureus and various laboratory and patient strains of MRSA are listed in Table 1. A partial listing of the antimicrobial activities are listed in Table 2 while a more complete table can be found in the ESI. $\dagger$ While a number of iridium complexes showed activity against $S$. aureus, two of those compounds, 4-Ir and 5-Ir were notable for their low MICs against both $S$. aureus and MRSA isolates (Table 2). Further, the MBCs for 5-Ir were almost the same as the MICs, demonstrating its bactericidal activity.

The data in Table 2 not only demonstrate that these complexes exhibit potent activity against both $S$. aureus and MRSA but also provide preliminary insight into the

Table 2 MICs (MBCs) of 1,2-diamine complexes of Cp complexes of iridium and cobalt against $S$. aureus and MRSA strains in $\mu \mathrm{g} \mathrm{mL}^{-1}$

\begin{tabular}{lllll}
\hline & $\begin{array}{l}\text { S. aureus } \\
\text { Compound }\end{array}$ & $\begin{array}{l}\text { MRSA } \\
\text { patient }\end{array}$ & $\begin{array}{l}\text { MRSA } \\
43300\end{array}$ & $\begin{array}{l}\text { MRSA } \\
34380\end{array}$ \\
\hline 1-Ir & $296(600)$ & $296(250)$ & $296(600)$ & $600(600)$ \\
2-Ir & $124(124)$ & $63(63)$ & $124(124)$ & $124(124)$ \\
3-Ir & $34(34)$ & $34(34)$ & $34(68)$ & $34(34)$ \\
4-Ir & $7(16)$ & $7(7)$ & $7(8)$ & $7(7)$ \\
5-Ir & $4(4)$ & $4(4)$ & $4(4)$ & $4(4)$ \\
6-Ir & $262(524)$ & $262(524)$ & $262(524)$ & $262(524)$ \\
7-Ir & $108(108)$ & $108(108)$ & $216(432)$ & $432(432)$ \\
8-Ir & $198(400)$ & $198(400)$ & $198(250)$ & $400(400)$ \\
9-Ir & $>500$ & $>500$ & $>500$ & $>500$ \\
10-Ir & $>500$ & $>500$ & $>500$ & $>500$ \\
11-Ir & $>500$ & $>500$ & $>500$ & $>500$ \\
12-Ir & $97(97)$ & $97(97)$ & $97(97)$ & $97(97)$ \\
13-Ir & $26(26)$ & $26(26)$ & $26(52)$ & $26(26)$ \\
14-Ir & $62.5(62.5)$ & $52(52)$ & $104(104)$ & $104(104)$ \\
15-Co & $11(22)$ & $22(22)$ & $44(44)$ & $44(44)$ \\
16-Co & $>500$ & $>500$ & $>500$ & $>500$ \\
Vanco & $0.7(0.7)$ & $0.7(0.7)$ & $0.7(0.7)$ & $0.7(0.7)$ \\
& & & &
\end{tabular}

structure-activity relationships (SARs) for these complexes. Our results thus far have suggested the following conclusions:

Substitutions to the amino groups with either four (9-Ir) or three (10-Ir) methyl groups on the nitrogen atoms leads to a loss of activity (Table 2). We speculate that the hydrogen bond contributions of the $\mathrm{NH}$-functionalities on opposite sides of the diamine core are important to activity.

Several observations support the conclusion that the active species in these experiments is the organometallic complex and not the metal-free ligand nor the ligand-free metal. First, all starting materials and complex intermediates were tested against the $S$. aureus panel and showed no activity. The fact that only some of these complexes display any activity against staph strains is good evidence that the complexes are not decomposing to form the metal ion or some other indiscriminate metal fragment - if that were the case all of the compounds would show activity. These observations lead us to conclude that these complexes are robust and do not dissociate under conditions of the assay to yield a common iridium or rhodium species that would account for activity.

Comparison between 1-Ir and 2-Ir where the only difference is the substitution on the $\mathrm{Cp}^{*}$ ring of an octyl group for one of the methyl groups shows an increase in activity of 2-Ir, the octyl substituted ring complex. Similarly, comparison between 3-Ir, 4-Ir and 5-Ir, all complexes with the cis-1,2diaminocyclohexane ligand, shows that there is an increase in activity for 4-Ir and 5-Ir where one methyl group of the Cp* ligand is substituted with either a hexyl or an octyl group. It is tempting to ascribe this effect to a simple increase in hydrophobicity of the complexes, but a more specific interaction may be at play. Evidence for this is that 8-Ir with the significantly longer dodecyl chain on the $\mathrm{Cp}^{*}$ ring has little activity.

Aryl groups on the ethylenediamine backbone also resulted in activity but the range of compounds is too small to draw any significant conclusions. However, greater activity is observed for 4-fluorophenyl groups, 13-Ir, than for 4-methoxyphenyl groups, 14-Ir.

The most dramatic difference in activity is observed for both iridium (3-Ir vs. 6-Ir) and cobalt (15-Co vs. 16-Co) where the sole difference is the use of the cis- vs. the trans-isomer of 1,2-diaminocyclohexane. Both isomers complex strongly with the iridium and cobalt, but only the complexes with the cis-isomer show significant activity against $S$. aureus.

\section{Time-kill measurements}

Survival of $S$. aureus strain ATCC 6358 and MRSA strain 34380 when exposed to $8 \mu \mathrm{g} \mathrm{mL} \mathrm{mL}^{-1}$ 4-Ir (i.e., MIC/MBC) after 1 , 2,3 , and $6 \mathrm{~h}$ exposure at $37^{\circ} \mathrm{C}$ were measured in two independent experiments (Fig. 1). 4-Ir killed both the antibioticsusceptible $S$. aureus and the methicillin-resistant $S$. aureus strains (Fig. 2). 


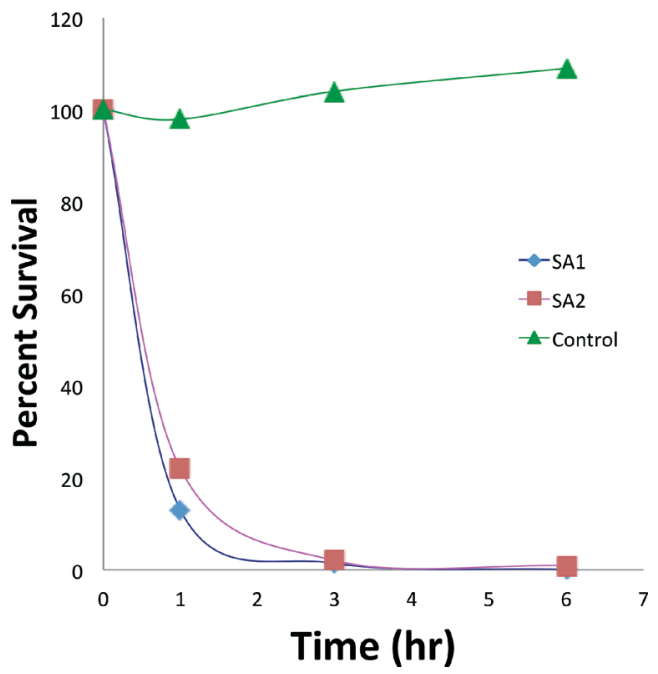

Fig. 1 Killing S. aureus strain ATCC 6358 with 4-Ir.

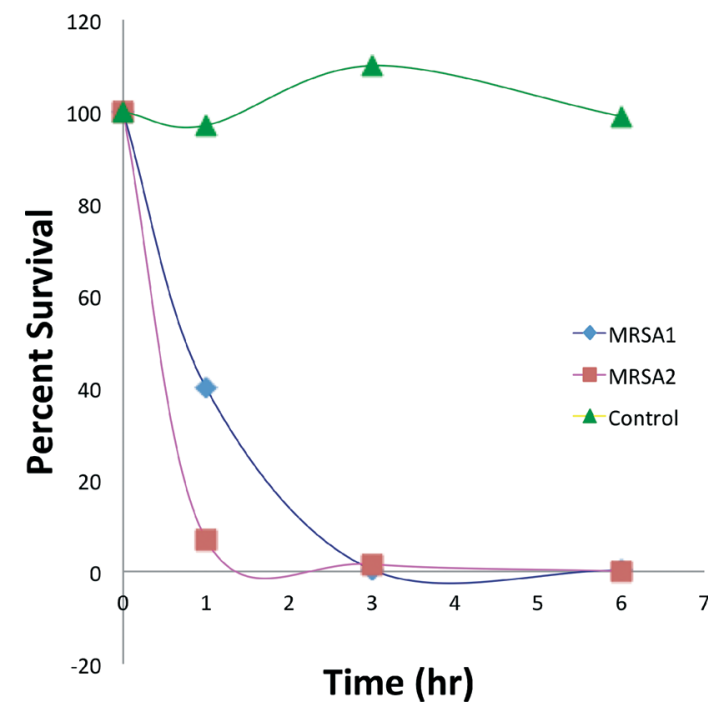

Fig. 2 Killing of MRSA strain 34380 with 4-Ir.

\section{Hemolytic activities}

The complexes with strong antibiotic activity (i.e., 3-Ir, 4-Ir, and 5-Ir) were tested for hemolytic activity ( $<10 \%$ hemolysis of sheep red blood cells). These complexes showed no hemolytic activity at their MICs or MBCs and hemolytic activity at the $10 \%$ level was not observed until concentrations of greater than $250 \mu \mathrm{g} \mathrm{mL} \mathrm{mere}^{-1}$ reached.

\section{Mammalian toxicity}

While the cobalt complexes discussed above were not tested, the iridium complexes that showed significant activity against $S$. aureus and MRSA strains showed no evidence of toxicity against Vero cell line ATCC CCL-81 (ref. 29) at the highest concentrations tested of $500 \mu \mathrm{g} \mathrm{mL} \mathrm{m}^{-1}$. In addition, compound 4-Ir was tested for toxicity in mice and it was determined that no detrimental toxicological effects were present at doses of $5 \mathrm{mg} \mathrm{kg}{ }^{-1} \cdot{ }^{29}$

\section{Experimental}

\section{Materials}

Unless otherwise stated, synthetic work was carried out in air with untreated solvents. Commercially available reagents including all diamines were obtained from Sigma-Aldrich, St. Louis, MO 63103. Deuterated solvents for NMR spectroscopy were obtained from Cambridge Isotope Laboratories.

\section{General procedure}

A round bottom flask was charged with appropriate amounts of the respective $\left[\mathrm{Cp}^{x \mathrm{R}} \mathrm{IrCl}_{2}\right]_{2}$ (ref. 9, 27, 30) in dichloromethane with magnetic stirring. After 10 minutes the 1,2-diamine was added in a 2.1 molar excess drop wise to the stirring solution. After the addition of the 1,2-diamine the solution was allowed to react for an additional 30 minutes. The complexes were recrystallized from dichloromethane and ether or hexanes and collected on a fine fritted filter as yellow crystalline powders. ${ }^{1} \mathrm{H}$ NMR and ${ }^{13} \mathrm{C}$ NMR spectra were collected on a Varian MR-400 NMR spectrometer. Highresolution mass spectrometry data was collected on Agilent 6220 Accurate Mass TOF LC-MS. Elemental analyses were performed by Atlantic Microlabs, Norcross, GA.

Synthesis of $\left[\left(\eta^{5}-\mathrm{C}_{5} \mathrm{Me}_{5}\right) \operatorname{Ir}(\right.$ ethylenediamine $\left.) \mathrm{Cl}\right] \mathrm{Cl}$ (1-Ir). Following the general procedure, $100 \mathrm{mg}(0.125 \mathrm{mmol})$ of $\left[\mathrm{Cp} * \mathrm{IrCl}_{2}\right]_{2}$ was combined with $15.9 \mathrm{mg}(0.264 \mathrm{mmol})$ of ethylenediamine in dichloromethane. Yield: $91.7 \mathrm{mg}(0.217$ mmol) (86\%) HRMS/ESI ${ }^{+}(\mathrm{m} / \mathrm{z})$ : calcd. for $\mathrm{C}_{12} \mathrm{H}_{23} \mathrm{~N}_{2}\left[{ }^{193} \mathrm{Ir}\right] \mathrm{Cl}$ 423.1257; found 423.1265. Anal calc. $\mathrm{C}_{12} \mathrm{H}_{23} \mathrm{Cl}_{2} \mathrm{~N}_{2} \mathrm{Ir} \mathrm{C}: 31.44$, $\mathrm{H}: 5.06$; found C: $31.65, \mathrm{H}: 5.10 .{ }^{1} \mathrm{H}$ NMR $\left(400 \mathrm{MHz}, \mathrm{CDCl}_{3}\right) \delta$ 6.81 (br s, 2H), 5.74 (br s, 2H), 3.27 (m, 2H), 3.07 (d, 2H), 1.89 (s, 15H, 5CpMe) ppm. ${ }^{13} \mathrm{C}$ NMR (101 MHz, $\left.\mathrm{CDCl}_{3}\right) \delta 93.2$ $(\mathrm{CpC}), 92.8(\mathrm{CpC}), 92.2(\mathrm{CpC}), 54.1\left(\mathrm{CH}_{2}\right) \mathrm{ppm}$.

Synthesis of $\left[\left(\eta^{5}-\mathrm{C}_{4} \mathrm{Me}_{4} \mathrm{C}_{8} \mathrm{H}_{17}\right) \operatorname{Ir}(\right.$ ethylenediamine $\left.) \mathrm{Cl}\right] \mathrm{Cl}$ (2Ir). Following the general procedure, $100 \mathrm{mg}(0.112 \mathrm{mmol})$ of $\left[\mathrm{Cp}^{*\left({ }^{(o c t y l}\right)} \mathrm{IrCl}_{2}\right]_{2}$ was combined with $14.2 \mathrm{mg}(0.235 \mathrm{mmol})$ of ethylenediamine in dichloromethane. Yield: $85.1 \mathrm{mg}(0.152$ mmol) (68\%) HRMS/ESI ${ }^{+}(m / z)$ : calcd. for $\mathrm{C}_{19} \mathrm{H}_{37} \mathrm{~N}_{2}\left[{ }^{193} \mathrm{Ir}\right] \mathrm{Cl}_{2}$ 556.1963; found 556.1959 ${ }^{1} \mathrm{H}$ NMR (400 $\mathrm{MHz} \mathrm{CDCl}_{3}$ ) $\delta 5.41$ (br s, 2H, NH), 4.65 (br s, 2H, NH), 3.35 (d, 2H, $\mathrm{CH}_{2}$ ), 3.07 (d, $\left.2 \mathrm{H}, \mathrm{CH}_{2}\right), 2.14(\mathrm{~m}, 2 \mathrm{H}), 1.96(\mathrm{~m}, 2 \mathrm{H}), 1.84$ (s, 15H, 5CpMe), 1.43 (m, 2H), 1.25 (br m, 4H), 1.05-0.96 (m, 4H), 0.84 (m, 3H, $\left.\mathrm{CH}_{3}\right)$ ppm.

Synthesis of $\left[\left(\eta^{5}-\mathrm{C}_{5} \mathrm{Me}_{5}\right) \operatorname{Ir}(\right.$ cis-1,2-diaminocyclohexane $\left.) \mathrm{Cl}\right]$ Cl (3-Ir). Following the general procedure, 87.1 $\mathrm{mg}(0.109$ $\mathrm{mmol})$ of $\left[\mathrm{IrCp}^{*} \mathrm{Cl}_{2}\right]_{2}$ was combined with $26.2 \mathrm{mg}(0.229$ $\mathrm{mmol})$ of cis-1,2-diaminocyclohexane. Yield: $100.7 \mathrm{mg}(0.211$ mmol) (96\%). HRMS/ESI ${ }^{+}(m / z)$ : calcd. for $\mathrm{C}_{16} \mathrm{H}_{29} \mathrm{~N}_{2}\left[{ }^{193} \mathrm{Ir}\right] \mathrm{Cl}$ 477.1747; found 477.1752. Anal calc. $\mathrm{C}_{16} \mathrm{H}_{28} \mathrm{Cl}_{2} \mathrm{~N}_{2} \operatorname{Ir}\left(\mathrm{H}_{2} \mathrm{O}\right) \mathrm{C}$ : 36.19, H: 5.71; found C 35.83, H: 5.73. ${ }^{1} \mathrm{H}$ NMR $(400 \mathrm{MHz}$, $\left.\mathrm{CDCl}_{3}\right) \delta 5.72\left(\mathrm{~s}, 2 \mathrm{H}, \mathrm{NH}_{2}\right), 3.27(\mathrm{~m}, 1 \mathrm{H}, \mathrm{CH}), 3.09(\mathrm{~m}, 1 \mathrm{H}$, $\mathrm{CH}), 2.34-2.14(\mathrm{~m}, 2 \mathrm{H}), 2.08(\mathrm{~d}, J=12.6 \mathrm{~Hz}, 2 \mathrm{H}), 1.91(\mathrm{~s}, 15 \mathrm{H}$, CpMe), 1.65 (q, $J=12.9 \mathrm{~Hz}, 2 \mathrm{H}, \mathrm{CH}_{2}$ ), 1.43 (q, $J=12.9 \mathrm{~Hz}$, 
2H), $1.26(\mathrm{~m}, 2 \mathrm{H}), 1.22\left(\mathrm{~m}, 2 \mathrm{H}, \mathrm{CH}_{2}\right) \mathrm{ppm} .{ }^{13} \mathrm{C}$ NMR (101 $\left.\mathrm{MHz}, \mathrm{CDCl}_{3}\right) \delta 93.53(\mathrm{CpC}), 92.25(\mathrm{CpC}), 84.75(\mathrm{Cp} C), 84.04$ $(\mathrm{CpC}), 34.9,32.05,31.72,28.14,26.93,21.41,10.44$, 9.74 ppm.

Synthesis of $\left[\left(\eta^{5}-\mathrm{C}_{4} \mathrm{Me}_{4} \mathrm{C}_{8} \mathrm{H}_{17}\right) \operatorname{Ir}(\right.$ cis-1,2-diaminocyclohexane $)$ Cl]Cl (4-Ir). Following the general procedure, $50.0 \mathrm{mg}$ $(0.056 \mathrm{mmol})$ of $\left[\mathrm{Cp}^{*(\text { octyl })} \mathrm{IrCl}_{2}\right]_{2}$ was combined with $24.3 \mathrm{mg}$ (0.212 mmol) of cis-1,2-diaminocyclohexane. Yield: $53.1 \mathrm{mg}(0.092 \mathrm{mmol})(83 \%)$. HRMS/ESI ${ }^{+}(\mathrm{m} / \mathrm{z})$ : calcd. for $\mathrm{C}_{23} \mathrm{H}_{43} \mathrm{~N}_{2}\left[{ }^{193} \mathrm{Ir}\right] \mathrm{Cl} 575.2751$; found 575.2764. Anal calc. $\mathrm{C}_{23} \mathrm{H}_{43} \mathrm{Cl}_{2} \mathrm{~N}_{2} \mathrm{Ir} \mathrm{C}: 45.23, \mathrm{H}: 7.10$; found $\mathrm{C}: 45.19, \mathrm{H}: 7.15 .{ }^{1} \mathrm{H}$ NMR (400 MHz, $\left.\mathrm{CDCl}_{3}\right) \delta 3.38(2, \mathrm{H}), 3.10(\mathrm{~m}, 2 \mathrm{H}), 2.24(\mathrm{t}$, $J=8.0 \mathrm{~Hz}, 4 \mathrm{H}), 2.08(\mathrm{~m}, 2 \mathrm{H}), 1.84$ (s, CpMe), 1.81 (s, CpMe), 1.78 (s, CpMe), 1.76 (s, CpMe) 1.64 (t, $J=8.0 \mathrm{~Hz}$, 2H, $\left.\mathrm{CH}_{2}\right), 1.43(\mathrm{p}, J=8.1 \mathrm{~Hz}, 4 \mathrm{H}), 1.32\left(\mathrm{~m}, 4 \mathrm{H}, 2 \mathrm{CH}_{2}\right), 1.16$ (m, 4H), 0.86 (t, 3H, CH $)$ ppm. ${ }^{13} \mathrm{C} \mathrm{NMR} \mathrm{(101} \mathrm{MHz,} \mathrm{CDCl}_{3}$ ) $\delta 88.2(\mathrm{CpC}), 86.2(\mathrm{CpC}) .86 .1(\mathrm{Cp} C), 51.39(\mathrm{CH}), 49.34$ $(\mathrm{CH}), 31.88\left(\mathrm{CH}_{2}\right), 31.87\left(\mathrm{CH}_{2}\right), 30.59\left(\mathrm{CH}_{2}\right), 29.69\left(\mathrm{CH}_{2}\right)$, $29.50\left(\mathrm{CH}_{2}\right), 29.28\left(\mathrm{CH}_{2}\right), 29.07\left(\mathrm{CH}_{2}\right), 27.61\left(\mathrm{CH}_{2}\right), 26.44$ $\left(\mathrm{CH}_{2}\right), 26.22\left(\mathrm{CH}_{2}\right), 25.17\left(\mathrm{CH}_{2}\right), 24.12\left(\mathrm{CH}_{2}\right), 15.25\left(\mathrm{CH}_{3}\right)$ ppm.

Synthesis of $\left[\left(\eta^{5}-\mathrm{C}_{4} \mathrm{Me}_{4} \mathrm{C}_{6} \mathrm{H}_{13}\right) \operatorname{Ir}(\right.$ cis-1,2-diaminocyclohexane) Cl]Cl (5-Ir). Following the general procedure, $50 \mathrm{mg}(0.057$ mmol) of $\left[\mathrm{IrCp}^{\left.*{ }^{(h e x y l}\right)} \mathrm{Cl}_{2}\right]_{2}$ was combined with $15.9 \mathrm{mg}$ (0.139 $\mathrm{mmol}$ ) of cis-1,2-diaminocyclohexane. Yield: $52.0 \mathrm{mg}$ (0.095 mmol) (85\%).HRMS/ESI ${ }^{+}(\mathrm{m} / \mathrm{z})$ : calcd. for $\mathrm{C}_{21} \mathrm{H}_{39} \mathrm{~N}_{2}\left[{ }^{193} \mathrm{Ir}\right] \mathrm{Cl}$ 547.2509; found 547.2541. Anal calc. C: 43.29, $\mathrm{H}$ : 6.75 ; found C: 43.078, H: 6.74. ${ }^{1} \mathrm{H}$ NMR $\left(400 \mathrm{MHz}, \mathrm{CDCl}_{3}\right) \delta 6.51(\mathrm{~s}, 1 \mathrm{H}$, $\mathrm{NH}), 5.71(\mathrm{~s}, 1 \mathrm{H}, \mathrm{NH}), 4.03(\mathrm{~m}, 1 \mathrm{H}, \mathrm{CH}), 3.31(\mathrm{~m}, 1 \mathrm{H}, \mathrm{CH})$, $2.24(\mathrm{t}, J=8.5,2 \mathrm{H}), 1.89-1.81(2 \mathrm{~s}, 15 \mathrm{H}, \mathrm{CpMe}), 1.78(\mathrm{~m}, 4 \mathrm{H})$, 1.47-1.34 (m, 4H), 1.34-1.12 (m, 2H), 0.92-0.76 (m, 3H, $\left.\mathrm{CH}_{3}\right)$ ppm. ${ }^{13} \mathrm{C}$ NMR (101 MHz, $\left.\mathrm{CDCl}_{3}\right) \delta 87.41(\mathrm{Cp} C), 86.14(\mathrm{Cp} C)$, $47.6(\mathrm{CH}), 46.31(\mathrm{CH}), 35.55\left(\mathrm{CH}_{2}\right), 32.64\left(\mathrm{CH}_{2}\right), 29.69\left(\mathrm{CH}_{2}\right)$, $29.50\left(\mathrm{CH}_{2}\right), 27.61\left(\mathrm{CH}_{2}\right), 26.44\left(\mathrm{CH}_{2}\right), 26.22,24.12\left(\mathrm{CH}_{2}\right)$, $12.45\left(\mathrm{CH}_{3}\right), 10.4$ (CpMe) ppm.

Synthesis of $\left[\left(\eta^{5}-\mathrm{C}_{5} \mathrm{Me}_{5}\right) \operatorname{Ir}( \pm\right.$-trans-1,2-diaminocyclohexane) Cl]Cl (6-Ir). Following the general procedure, $47.1 \mathrm{mg}$ (0.059 $\mathrm{mmol})$ of $\left[\mathrm{Cp}^{*} \mathrm{IrCl}_{2}\right]_{2}$ was combined with $15.2 \mathrm{mg}$ (0.133 $\mathrm{mmol})$ of trans-1,2-diaminocyclohexane. Yield $48.1 \mathrm{mg}$ (0.101 mmol) (81\%). Calcd. for $\mathrm{C}_{16} \mathrm{H}_{29} \mathrm{~N}_{2}\left[{ }^{193} \mathrm{Ir}\right] \mathrm{Cl}$ 477.1765; found 477.1761. ${ }^{1} \mathrm{H}$ NMR (400 MHz, $\mathrm{CDCl}_{3}$ ) $\delta 5.68\left(\mathrm{~s}, 2 \mathrm{H}, \mathrm{NH}_{2}\right) 3.27$ (m, 1H, CH), $3.15(\mathrm{~m}, 1 \mathrm{H}, \mathrm{CH}), 2.25(\mathrm{~m}, 2 \mathrm{H}), 2.08$ (d, $J=11.6$ $\mathrm{Hz}, 2 \mathrm{H}$ ), 1.84 (2s, 15H, CpMe), 1.65 (q, $J=11.6 \mathrm{~Hz}, 2 \mathrm{H}, \mathrm{CH}_{2}$ ), $1.43(\mathrm{q}, J=12.9 \mathrm{~Hz}, 2 \mathrm{H}) 1.26(\mathrm{~m}, 2 \mathrm{H}), 1.22\left(\mathrm{~m}, 2 \mathrm{H}, \mathrm{CH}_{2}\right)$ ppm. ${ }^{13} \mathrm{C}$ NMR (101 MHz, $\left.\mathrm{CDCl}_{3}\right) \delta 94.85(\mathrm{Cp} C), 93.25(\mathrm{CpC})$, $84.75(\mathrm{CpC}), 84.04(\mathrm{CpC}), 34.9,32.05,30.82,28.14,27.33$, 21.41, (cyclohexane) 10.18 (CpMe) ppm.

Synthesis of $\left[\left(\eta^{5}-\mathrm{C}_{4} \mathrm{Me}_{4} \mathrm{C}_{8} \mathrm{H}_{17}\right) \operatorname{Ir}(\right.$ trans- $\pm-1,2$-diaminocyclohexane $)$ Cl]Cl (7-Ir). Following the general procedure, $75.0 \mathrm{mg}(0.083$ $\mathrm{mmol})$ of $\left[\mathrm{Cp}^{*(\mathrm{octyl})} \mathrm{IrCl}_{2}\right]_{2}$ was combined with $36.4 \mathrm{mg}(0.318$ $\mathrm{mmol})$ of trans-1,2-diaminocyclohexane. Yield: $71.0 \mathrm{mg}(0.119$ mmol) (65\%). HRMS/ESI ${ }^{+}(\mathrm{m} / \mathrm{z})$ : calcd for $\mathrm{C}_{23} \mathrm{H}_{43} \mathrm{~N}_{2}\left[{ }^{193} \mathrm{Ir}\right] \mathrm{Cl}$ 575.2755; found 575.2771. ${ }^{1} \mathrm{H}$ NMR (400 $\left.\mathrm{MHz}, \mathrm{CDCl}_{3}\right) \delta 3.56$ $\left(2, \mathrm{NH}_{2}\right), 3.36(\mathrm{~m}, 2 \mathrm{H}), 2.75(\mathrm{t}, J=9.5 \mathrm{~Hz}, 4 \mathrm{H}), 2.17(\mathrm{~m}, 2 \mathrm{H})$, 1.86 (s, CpMe), 1.84 (s, CpMe), 1.81 (s, CpMe), 1.76 (s, СрMе), $1.64\left(\mathrm{t}, J=9.5 \mathrm{~Hz}, 2 \mathrm{H}, \mathrm{CH}_{2}\right), 1.43$ (p, $\left.J=8.1 \mathrm{~Hz}, 4 \mathrm{H}\right), 1.37(\mathrm{~m}$,
4H, 2CH $\left.\mathrm{CH}_{2}\right), 1.16(\mathrm{~m}, 4 \mathrm{H}), 0.91\left(\mathrm{t}, 3 \mathrm{H}, \mathrm{CH}_{3}\right) \mathrm{ppm} .{ }^{13} \mathrm{C} \mathrm{NMR}$ $\left(101 \mathrm{MHz}, \mathrm{CDCl}_{3}\right) \delta 88.7(\mathrm{CpC}), 88.5(\mathrm{CpC}), 84.2(\mathrm{CpC}), 51.56$ $(\mathrm{CH}), 49.65(\mathrm{CH}), 32.88\left(\mathrm{CH}_{2}\right), 30.77\left(\mathrm{CH}_{2}\right), 30.59\left(\mathrm{CH}_{2}\right), 29.69$ $\left(\mathrm{CH}_{2}\right), 29.50\left(\mathrm{CH}_{2}\right), 29.28\left(\mathrm{CH}_{2}\right), 29.07\left(\mathrm{CH}_{2}\right), 26.81\left(\mathrm{CH}_{2}\right)$, $26.44\left(\mathrm{CH}_{2}\right), 25.44\left(\mathrm{CH}_{2}\right), 25.17\left(\mathrm{CH}_{2}\right), 24.12\left(\mathrm{CH}_{2}\right), 15.18$ $\left(\mathrm{CH}_{3}\right)$ ppm.

Synthesis of $\left[\left(\eta^{5}-\mathrm{C}_{4} \mathrm{Me}_{4} \mathrm{C}_{12} \mathrm{H}_{25}\right) \operatorname{Ir}(\right.$ cis-1,2-diaminocyclohexane) Cl]Cl (8-Ir). Following the general procedure, $50.0 \mathrm{mg}(0.051$ $\mathrm{mmol})$ of $\left[\mathrm{Cp}^{*(\text { dodecyl })} \mathrm{IrCl}_{2}\right]_{2}$ was combined with $12.0(0.105$ $\mathrm{mmol}) \mathrm{mg}$ of cis-1,2-diaminocyclohexane. Yield: $39.2 \mathrm{mg}(0.062$ mmol) (60\%). HRMS/ESI ${ }^{+}(\mathrm{m} / z)$ : calcd. for $\mathrm{C}_{27} \mathrm{H}_{51} \mathrm{~N}_{2}\left[{ }^{193} \mathrm{Ir}\right] \mathrm{Cl}$ 631.3448; found 631.3475. ${ }^{1} \mathrm{H}$ NMR (400 MHz, $\left.\mathrm{CDCl}_{3}\right) \delta 5.69$ (s, 1H, NH), $4.24(\mathrm{~s}, 1 \mathrm{H}, \mathrm{NH}), 3.24(\mathrm{~m} .1 \mathrm{H}, \mathrm{CH}), 3.05(\mathrm{~m}, 1 \mathrm{H}$, $\mathrm{CH})$, 2.39-2.19 (bm, 6H), 1.85 (s, 15H, CpMe), 1.71-1.62 (m, $4 \mathrm{H})$, 1.61-1.52 (m, 4H), 1.51-1.45 (m, 4H), 1.40 (m, 6H), 1.35-1.15 (m, 10H), 0.84 (m, 3H, $\left.\mathrm{CH}_{3}\right)$ ppm.

Synthesis of $\left[\left(\eta^{5}-C_{5} M_{5}\right) \operatorname{Ir}\left(N, N, N^{\prime}, N^{\prime}\right.\right.$-tetramethyl)ethylenediamine $)$ Cl]Cl (9-Ir). Following the general procedure, $50.0 \mathrm{mg}(0.062$ $\mathrm{mmol})$ of $\left[\mathrm{Cp}^{*} \mathrm{IrCl}_{2}\right]_{2}$ was combined in dichloromethane with $18.1 \mathrm{mg}(0.155 \mathrm{mmol})$ of $N, N, N^{\prime}, N^{\prime}$-tetramethylethylenediamine. Yield: $42.2 \mathrm{mg}$ (0.088 mmol). $\mathrm{HRMS} \mathrm{ESI}^{+}(\mathrm{m} / \mathrm{z})$ : calcd. for $\mathrm{C}_{16} \mathrm{H}_{31} \mathrm{~N}_{2}\left[{ }^{193} \mathrm{Ir}\right] \mathrm{Cl} \quad 479.1805$; found 479.1804. ${ }^{1} \mathrm{H} \quad \mathrm{NMR}$ $\left(400 \mathrm{MHz} \mathrm{CDCl}_{3}\right) \delta 3.62\left(\mathrm{~m}, 3 \mathrm{H}, \mathrm{CH}_{3}\right), 3.23\left(\mathrm{~s}, 3 \mathrm{H}, \mathrm{CH}_{3}\right), 2.88$ (m, 3H, $\left.\mathrm{CH}_{3}\right), 2.42\left(\mathrm{~m}, 2 \mathrm{H}, \mathrm{CH}_{2}\right) 1.89$ (s, 15H, CpMe) ppm.

Synthesis of $\left[\left(\eta^{5}-\mathrm{C}_{5} \mathrm{Me}_{5}\right) \operatorname{Ir}\left(\boldsymbol{N}, N, N^{\prime}\right.\right.$-trimethyl)ethylenediamine $)$ Cl]Cl (10-Ir). Following the general procedure, $50.0 \mathrm{mg}(0.062$ $\mathrm{mmol})$ of $\left[\mathrm{Cp}^{*} \mathrm{IrCl}_{2}\right]_{2}$ was combined in dichloromethane with $17.8 \mathrm{mg}(0.174 \mathrm{mmol})$ of $N, N, N^{\prime}$-trimethylethylenediamine dissolved in $10 \mathrm{~mL}$ of methanol. Yield: $36.2 \mathrm{mg}$ (0.078 mmol). HRMS/ESI ${ }^{+}(\mathrm{m} / \mathrm{z})$ : calcd. for $\mathrm{C}_{15} \mathrm{H}_{29} \mathrm{~N}_{2}\left[{ }^{193} \mathrm{Ir}\right] \mathrm{Cl}$ 465.1648; found 465.1650. ${ }^{1} \mathrm{H}$ NMR (400 MHz, $\mathrm{CDCl}_{3}$ ) $\delta 4.65$ (br s, $1 \mathrm{H}, \mathrm{NH}$ ), 3.42 $\left(\mathrm{m}, 3 \mathrm{H}, \mathrm{CH}_{3}\right), 3.12\left(\mathrm{~m}, 2 \mathrm{H}, \mathrm{CH}_{2}\right), 2.65\left(\mathrm{~m}, 3 \mathrm{H}, \mathrm{CH}_{3}\right), 1.87$ (s, 15H, CpMe) ppm.

Synthesis of $\left[\left(\eta^{5}-\mathrm{C}_{5} \mathrm{Me}_{5}\right) \operatorname{Ir}(N-(1-\right.$ benzyl $)$ ethylenediamine $)$ $\mathrm{Cl}][\mathrm{Cl}]$ (11-Ir). Following the general procedure: $50.0 \mathrm{mg}$ $(0.062 \mathrm{mmol})$ of $\left[\mathrm{Cp}^{*} \mathrm{IrCl}_{2}\right]_{2}$ was combined in dichloromethane with $19.8 \mathrm{mg}(0.171 \mathrm{mmol})$ of $N$-(1-benzyl)ethylenediamine dissolved in $10 \mathrm{~mL}$ of methanol. Yield: $42.3 \mathrm{mg}(0.082$ mmol). HRMS/ESI ${ }^{+}(\mathrm{m} / \mathrm{z})$ : calcd. for $\mathrm{C}_{19} \mathrm{H}_{29} \mathrm{~N}_{2}\left[{ }^{193} \mathrm{Ir}\right] \mathrm{Cl}$ 513.1649; found 513.1648. ${ }^{1} \mathrm{H}$ NMR (400 $\left.\mathrm{MHz} \mathrm{CDCl}_{3}\right) \delta 7.39$ (m, 4H, CH) 7.26 (d, 1H, CH), 5.68 (d, 2H, NH), $5.52(\mathrm{~s}, 1 \mathrm{H}$, $\mathrm{NH}$ ), 4.57 (s, 2H), 2.1 (br s, 2H), 1.73 (s, 15H, CpMe), 1.35 (s, 2H) ppm. ${ }^{13} \mathrm{C}$ NMR (101 MHz, $\mathrm{CDCl}_{3}$ ) $\delta 125.6$ (aryl), 124.9 (aryl), $88.2(\mathrm{CpC}), 87.1(\mathrm{CpC}), 55.2\left(\mathrm{CH}_{2}\right), 51.4\left(\mathrm{CH}_{2}\right), 12.5$ $\left(\mathrm{CH}_{3}\right)$ ppm.

Synthesis of $\left[\left(\eta^{5}-\mathrm{C}_{5} \mathrm{Me}_{4} \mathrm{C}_{8} \mathrm{H}_{17}\right) \operatorname{Ir}(N\right.$-(1-naphthyl)ethylenediamine) Cl]Cl (12-Ir). Following the general procedure, $50.0 \mathrm{mg}(0.056$ $\mathrm{mmol})$ of $\left[\mathrm{Cp}^{*(\text { octyl })} \mathrm{IrCl}_{2}\right]_{2}$ was combined in dichloromethane with $32.1 \mathrm{mg}(0.171 \mathrm{mmol})$ of $N$-(1-napthyl)ethylenediamine dissolved in $10 \mathrm{~mL}$ of methanol. Yield: $40.6 \mathrm{mg}(0.062 \mathrm{mmol})$ (55\%). HRMS/ESI ${ }^{+}(\mathrm{m} / \mathrm{z})$ : calcd. for $\mathrm{C}_{29} \mathrm{H}_{43} \mathrm{~N}_{2}\left[{ }^{193} \mathrm{Ir}\right]$ 647.2321; found 647.2394. Anal calc. $\mathrm{C}_{29} \mathrm{H}_{43} \mathrm{Cl}_{2} \mathrm{~N}_{2} \mathrm{Ir} \mathrm{C}: 49.71, \mathrm{H}: 6.46$; found C: 48.86, H: 5.96. ${ }^{1} \mathrm{H}$ NMR (400 $\left.\mathrm{MHz}, \mathrm{CDCl}_{3}\right) \delta 7.55$ (d, $J=6.9 \mathrm{~Hz}, 1 \mathrm{H}, \operatorname{Ar} H), 7.41(\mathrm{~d}, J=6.9 \mathrm{~Hz}, 1 \mathrm{H}, \operatorname{Ar} H), 7.14(\mathrm{~m}$, $4 \mathrm{H}, \operatorname{Ar} H), 4.88(\mathrm{~m}, 2 \mathrm{H}), 3.72(\mathrm{~d}, J=7.5 \mathrm{~Hz}, 1 \mathrm{H}), 3.60(\mathrm{~d}, J=$ $7.5 \mathrm{~Hz}, 1 \mathrm{H}), 3.11(\mathrm{~s}, 2 \mathrm{H}), 2.36(\mathrm{t}, J=11.5 \mathrm{~Hz}, 2 \mathrm{H}), 2.02(\mathrm{~s}, 3 \mathrm{H}$, 
CpMe), 1.99 (s, 3H, CpMe) 1.93 (s, 3H, CpMe), 1.89 (s, 3H, CpMe), 1.79 (t, $J=12.6,2 \mathrm{H}, \mathrm{CH}_{2}$ ), 1.72 (d, 1H, CH), 1.52-1.28 $(\mathrm{m}, 4 \mathrm{H}), 1.16\left(\mathrm{~m}, J=11.5 \mathrm{~Hz}, 3 \mathrm{H}, \mathrm{CH}_{3}\right) \mathrm{ppm} .{ }^{13} \mathrm{C} \mathrm{NMR}$ $\left(101 \mathrm{MHz}, \mathrm{CDCl}_{3}\right.$ ) $\delta 129.9$ (aryl), 127.9 (aryl), 127.8 (aryl), $86.2(\mathrm{CpC}), 86.1(\mathrm{CpC}), 60.39\left(\mathrm{CH}_{2}\right), 58.2\left(\mathrm{CH}_{2}\right), 31.88\left(\mathrm{CH}_{2}\right)$, $31.87\left(\mathrm{CH}_{2}\right), 30.59\left(\mathrm{CH}_{2}\right), 35.69\left(\mathrm{CH}_{2}\right), 32.50\left(\mathrm{CH}_{2}\right), 27.28$ $\left(\mathrm{CH}_{2}\right), 26.07\left(\mathrm{CH}_{2}\right), 27.55\left(\mathrm{CH}_{2}\right), 22.30\left(\mathrm{CH}_{2}\right), 14.15\left(\mathrm{CH}_{3}\right)$ ppm.

Synthesis of $\left[\left(\eta^{5}-\mathrm{C}_{5} \mathrm{Me}_{5}\right) \operatorname{Ir}(1 S, 2 S)-1,2-\mathrm{bis}(4-\right.$ fluorophenyl)ethylenediamineCl]Cl (13-Ir). Following the general procedure, $50.0 \mathrm{mg}(0.062 \mathrm{mmol})$ of $\left[\mathrm{Cp}^{*} \mathrm{IrCl}_{2}\right]_{2}$ was combined with $42.3 \mathrm{mg} \quad(0.131 \mathrm{mmol}) \quad(1 S, 2 S)$-1,2-bis(4-fluorophenyl)ethylenediamine dihydrochloride in a 50/50 mix of dichloromethane and methanol. $15 \mathrm{mg}$ of sodium bicarbonate was added to the solution mixture. Yield: $67.5 \mathrm{mg}(0.110$ mmol) (88\%) HRMS/ESI ${ }^{+}(m / z)$ : calcd. for $\mathrm{C}_{24} \mathrm{H}_{29} \mathrm{~N}_{2} \mathrm{~F}_{2}\left[{ }^{193} \mathrm{Ir}\right] \mathrm{Cl}$ 611.1622; found 611.1636. Anal calc. C: 44.51, H: 4.58; found C: 44.58, H: 4.67. ${ }^{1} \mathrm{H}$ NMR (400 MHz, DMSO- $\left.d_{6}\right) 7.29$ (dd, $J=$ 8.5, $5.5 \mathrm{~Hz}, 2 \mathrm{H}$ ), 7.22 (dd, $J=8.5,5.5 \mathrm{~Hz}, 2 \mathrm{H}), 7.10-7.02$ (m, $4 \mathrm{H}), 6.81(\mathrm{~m}, 1 \mathrm{H}), 6.24-6.12(\mathrm{~m}, 1 \mathrm{H}, \mathrm{NH}), 5.58(\mathrm{~m}, 1 \mathrm{H}, \mathrm{NH})$, $4.61(\mathrm{t}, J=11.5 \mathrm{~Hz}, 1 \mathrm{H}), 4.40(\mathrm{t}, J=11.5,1 \mathrm{H}), 1.72(\mathrm{~s}, 15 \mathrm{H}$, CpMe) ppm. ${ }^{13} \mathrm{C}$ NMR (101 MHz, DMSO- $\left.d_{6}\right) 144.8$ (aryl), 135.1 (aryl), 132.5 (aryl), 129.7 (aryl), $85.8(\mathrm{CpC}), 26.7(\mathrm{CH})$, 8.95 (CpMe) ppm.

Synthesis of $\quad\left[\left(\eta^{5}-\mathrm{C}_{5} \mathrm{Me}_{4} \mathrm{C}_{8} \mathrm{H}_{17}\right) \operatorname{Ir}(1 S, 2 S)-1,2-b i s(4-\right.$ methoxyphenyl)ethylenediamine]Cl (14-Ir). Following the general procedure, $50.0 \mathrm{mg}(0.056 \mathrm{mmol})$ of $\left[\mathrm{IrCp}^{*} \mathrm{Cl}_{2}\right]_{2}$ was combined with $46.2 \mathrm{mg}(0.145 \mathrm{mmol}$ of $(1 S, 2 S)$-1,2-bis(4methoxyphenyl)ethylenediamine dihydrochloride in a 50/50 mix of dichloromethane and methanol. $17 \mathrm{mg}$ of sodium bicarbonate was added to the solution mixture. Yield $48.3 \mathrm{mg}$ (0.076 mmol (67\%). HRMS/ESI ${ }^{+}(\mathrm{m} / \mathrm{z})$ : calcd. for $\mathrm{C}_{26} \mathrm{H}_{35} \mathrm{~N}_{2} \mathrm{O}_{2}\left[{ }^{193} \mathrm{Ir}\right] \mathrm{Cl}$ 635.2008; found: 635.2005. ${ }^{1} \mathrm{H}$ NMR (400 $\left.\mathrm{MHz}, \mathrm{CDCl}_{3}\right) \delta 7.49(\mathrm{~d}, J=8.5 \mathrm{~Hz}, 1 \mathrm{H}), 7.38(\mathrm{~d}, J=8.5 \mathrm{~Hz}$, $1 \mathrm{H}), 6.71(\mathrm{~d}, J=8.6 \mathrm{~Hz}, 1 \mathrm{H}), 6.62(\mathrm{~d}, \mathrm{~J}=8.6 \mathrm{~Hz}, 1 \mathrm{H}), 4.79(\mathrm{~m}$, $1 \mathrm{H}, \mathrm{NH}), 3.67(\mathrm{~m}, 2 \mathrm{H}), 3.55(\mathrm{~m}, 2 \mathrm{H}), 2.01(\mathrm{~s}, 2 \mathrm{H}), 1.88$ (s, 15H, CpMe), 1.59 (s, 6H, 2CH $\mathrm{CH}_{3}$ ppm. ${ }^{13} \mathrm{C}$ NMR (101 MHz, $\left.\mathrm{CDCl}_{3}\right) \delta 159.56$ (aryl), 130.24 (aryl), 129.78 (aryl), 129.60 (aryl), 114.32 (aryl), 114.16 (aryl), $86.68(\mathrm{Cp} C), 77.19(\mathrm{CpC})$, $63.27(\mathrm{CH}), 55.11(\mathrm{CH}), 54.99(\mathrm{CH}), 9.52$ (CpMe), 9.31 (СpMe) ppm.

Synthesis of $\left[\left(\eta^{5}-\mathrm{C}_{5} \mathbf{M e}_{5}\right) \operatorname{Ir}(\right.$ ethambutol $\left.) \mathrm{Cl}\right][\mathrm{Cl}]$. Following the general procedure, $100 \mathrm{mg}(0.125 \mathrm{mmol})$ of $\left[\mathrm{Cp}^{*} \mathrm{IrCl}_{2}\right]_{2}$ was combined with $72.8 \mathrm{mg}(0.262 \mathrm{mmol})$ ethambutol dihydrochloride in a 50/50 mix of dichloromethane and methanol. $20 \mathrm{mg}$ of sodium bicarbonate was added to the solution mixture. Yield: $67.7 \mathrm{mg}$ (0.113 mmol) (45\%). HRMS/ $\mathrm{ESI}^{+}(\mathrm{m} / \mathrm{z})$ : calcd. for $\mathrm{C}_{20} \mathrm{H}_{39} \mathrm{~N}_{2} \mathrm{O}_{2}\left[{ }^{193} \mathrm{Ir}\right] \mathrm{Cl}_{2}$ 602.2018; found 602.2022. ${ }^{1} \mathrm{H}$ NMR (400 MHz, $\left.\mathrm{D}_{2} \mathrm{O}\right) \delta 4.13$, (m, 4H) $3.23(\mathrm{~m}$, 2H), 2.21 (m, 2H, 2CH), 1.86 (s, 15H, CpMe), 1.21 (m, 3H) ppm.

Synthesis of $\left[\left(\eta^{5}-\mathrm{C}_{5} \mathrm{H}_{5}\right) \mathrm{Co}(\right.$ cis-1,2-diaminocylohexane $\left.) \mathrm{I}\right] \mathrm{I}$ (15-Co). $100 \mathrm{mg}(0.240 \mathrm{mmol})$ of $\mathrm{CpCo}(\mathrm{CO}) \mathrm{I}_{2}$ was dissolved in dichloromethane and allowed to stir for 10 minutes under nitrogen. 2.1 molar equivalents of cis-1,2-diaminocyclohexane was added dropwise via syringe to the solution and allowed to stir for 20 minutes under nitrogen. The solution immediately evolved CO gas from the reaction. The solution turned a clear red-brown color. The solvent and excess cis-1,2-diaminocyclohexane were removed using a rotary evaporator leaving a dark red-brown powder. The powder was then dissolved in deionized water and filtered on a fine frit. The filtrate was collected and the water was removed to yield $76 \mathrm{mg}(0.208$ mmol) $86 \%$. HRMS/ESI ${ }^{+}(\mathrm{m} / \mathrm{z})$ : calcd. for $\mathrm{C}_{11} \mathrm{H}_{19} \mathrm{~N}_{2}$ [Co]I 365.0033; found 364.9924. ${ }^{1} \mathrm{H}$ NMR (400 MHz, methanol- $d$ ) $\delta$ 4.8 (s, H, Cp), $3.29(\mathrm{~m}, 2 \mathrm{H}, 2 \mathrm{CH}), 1.79\left(\mathrm{~m}, 4 \mathrm{H}, 2 \mathrm{CH}_{2}\right), 1.35$ $\left(\mathrm{m}, 4 \mathrm{H}, 2 \mathrm{CH}_{2}\right), 1.6(\mathrm{~m}, 4 \mathrm{H}), 1.35\left(\mathrm{~m}, 4 \mathrm{H}, 2 \mathrm{CH}_{2}\right) \mathrm{ppm}$.

Following the same synthetic route as the previous cobalt complex, \pm -trans-1,2-diaminocyclohexane was used to synthesize $\left[\left(\eta^{5}-\mathrm{C}_{5} \mathrm{H}_{5}\right) \mathrm{Co}( \pm\right.$-trans-1,2-diaminocylohexane $\left.) \mathrm{I}\right] \mathrm{I}$ (16-Co). Yield $67 \mathrm{mg}(0.184 \mathrm{mmol})$ 76\%. HRMS/ESI ${ }^{+}(\mathrm{m} / \mathrm{z})$ : calcd. for $\mathrm{C}_{11} \mathrm{H}_{19} \mathrm{~N}_{2}$ [Co]I 365.0041; found 365.0052. ${ }^{1} \mathrm{H}$ NMR (400 MHz, methanol-d) $\delta 5.18(\mathrm{~s}, \mathrm{H}, \mathrm{Cp}), 3.41(\mathrm{~m}, 2 \mathrm{H}, 2 \mathrm{CH}), 1.82(\mathrm{~m}$, $\left.4 \mathrm{H}, 2 \mathrm{CH}_{2}\right), 1.42\left(\mathrm{~m}, 4 \mathrm{H}, 2 \mathrm{CH}_{2}\right), 1.53(\mathrm{~m}, 4 \mathrm{H}), 1.44(\mathrm{~m}, 4 \mathrm{H}$, $\left.2 \mathrm{CH}_{2}\right) \mathrm{ppm}$.

\section{Staphylococcal strains and measurement of MIC and MBC}

Staphylococcus aureus strains ATCC 6358 and ATCC 29213 were obtained from the American Type Culture Collection (ATCC), the unrelated methicillin-resistant $S$. aureus strain 43330 was obtained from Danville Community Hospital, and the recent patient isolates of MRSA (523000, 522870, 34864, 36361, 53016, and 34380) were obtained from Georgetown University Medical Center. ${ }^{31}$ MICs were measured by broth microdilution of fresh overnight cultures according to the Clinical and Laboratory Standards Institute (CCLI) guidelines with cation-adjusted Mueller-Hinton broth and an inoculum of $10^{5} \mathrm{CFU} \mathrm{mL}{ }^{-1} \cdot{ }^{31}$ Stocks of the compounds were dissolved in Mueller-Hinton broth. The MIC $\left(\mu \mathrm{g} \mathrm{mL}{ }^{-1}\right.$ ) was defined as the lowest concentration of compound completely inhibiting the appearance of turbidity by eye and confirmed by absorbance $540 \mathrm{~nm}$. The MBC ( $\mu \mathrm{g} \mathrm{mL} \mathrm{m}^{-1}$ ) was defined as the lowest concentration of compound reducing the colony count by $99.9 \%$ of the colony count in the initial, compound-free, inoculated well after $24 \mathrm{hr}$ incubation. All results represent the average of three independent measurements. Both MIC and MBC experiments were also performed using brain heart infusion broth (BHIB), resulting in similar data for both $\mathrm{MIC}$ and $\mathrm{MBC}^{32}$

\section{Time-kill measurements}

A single colony of the strain was inoculated into $2 \mathrm{~mL}$ of Mueller-Hinton broth in a $16 \times 125 \mathrm{~mm}$ screw cap tube and incubated overnight at $37{ }^{\circ} \mathrm{C}$ without aeration. One $\mathrm{mL}$ of that culture was inoculated into $9 \mathrm{~mL}$ of cation-adjusted Mueller-Hinton broth in a $125 \mathrm{~mL}$ flask and the culture incubated at $37{ }^{\circ} \mathrm{C}$ with aeration $(60 \mathrm{rpm})$ until reaching mid-log phase $\left(2 \times 10^{8} \mathrm{CFU} \mathrm{mL}{ }^{-1}\right)$. A dilution of that culture was then added to $20 \mathrm{~mL}$ of Mueller-Hinton broth to reach a final density of $10^{5} \mathrm{CFU} \mathrm{mL}^{-1}$. A volume of the complex stock in Mueller-Hinton broth was added to the S. aureus or MRSA suspension to reach its MIC and immediately and at 1, 3, 
and $6 \mathrm{hr}$, a $1 \mathrm{~mL}$ sample was removed and surviving CFU $\mathrm{mL}^{-1}$ were measured by spreading $0.1 \mathrm{~mL}$ (in triplicate) of undiluted, $10^{-1}, 10^{-2}$, and $10^{-3}$ fold diluted suspensions on Mueller-Hinton agar and incubating plates at $37{ }^{\circ} \mathrm{C}$ for 1 day. The results are reported as percent (\%) surviving cells at each time point from two independent measurements. ${ }^{32}$

\section{Toxicity studies}

Adapting the protocol of Babiceanu et al., ${ }^{33}$ the iridium complexes were tested for toxicity against Vero cell line ATC CCL81. While the cobalt complexes were not tested, the iridium compounds studied showed no toxicity at the maximum level tested of $500 \mu \mathrm{g} \mathrm{mL} \mathrm{m}^{-1}$.

\section{Hemolysis measurements}

Hemolysis measurements were performed as described in Maisuria et al. $^{31}$ and results reported at the lowest concentration of compound yielding $10 \%$ hemolysis $\left(\mathrm{IC}_{10 \%}\right)$.

\section{Conclusions}

The transition metal complexes described here show specific activity against $S$. aureus strains and lack activity against other microorganisms (i.e., Escherichia coli, Mycobacterium smegmatis, Candida albicans, and Aspergillus niger). In addition, the specificity of the complexes that show activity indicates that there are specific structure/activity relationships that must be met. This is most dramatically noted in the high activity observed for complexes of the cis isomer of 1,2diaminocyclohexane (3-Ir and 15-Co) and the total lack of activity of the complexes made with the trans isomer of 1,2diaminocyclohexane (6-Ir and 16-Co). We have begun work on elucidating the mechanism of action of this novel class of anti-microbial agents and hope to report on those findings in the near future. The combination of high and specific activity for certain complexes, the low cytotoxicity as tested with Vero cells and the low hemolytic activity all suggest that this transition metal platform may prove to be useful in overcoming antibiotic resistance in $S$. aureus.

\section{Acknowledgements}

The authors acknowledge the guidance and technical assistance of Ms. Myra D. Williams with the preparation of cultures and measurement of MICs and MBCs and Ms. Chelsea Dollarhite with the measurement of killing and David DePena for aiding in the synthesis of compounds. They also acknowledge a grant of syringes from the Hamilton Company.

\section{Notes and references}

1 R. M. Klevens, M. A. Morrison, J. Nadle, S. Petit, K. Gershman, S. Ray, L. H. Harrison, R. Lynfield, G. Dumyati and J. M. Townes, JAMA, 2007, 298, 1763-1771.

2 M. E. De Kraker, P. G. Davey, H. Grundmann and B. S. Group, PLoS Med., 2011, 8, e1001104.
3 J. L. Hadler, S. Petit, M. Mandour and M. L. Cartter, Emerging Infect. Dis., 2012, 18, 917.

4 I. Naseri, R. C. Jerris and S. E. Sobol, Arch. Otolaryngol., Head Neck Surg., 2009, 135, 14-16.

5 S. Saxena, P. Thompson, R. Birger, A. Bottle, N. Spyridis, I. Wong, A. P. Johnson, R. Gilbert and M. Sharland, Emerging Infect. Dis., 2010, 16, 530-533.

6 R. Kouyos, E. Klein and B. Grenfell, PLoS Pathog., 2013, 9, e1003134.

7 N. Balaban, O. Cirioni, A. Giacometti, R. Ghiselli, J. B. Braunstein, C. Silvestri, F. Mocchegiani, V. Saba and G. Scalise, Antimicrob. Agents Chemother., 2007, 51, 2226-2229.

8 J. C. Dabrowiak, Metals in medicine, John Wiley \& Sons, 2009.

9 Z. Liu, A. Habtemariam, A. M. Pizarro, S. A. Fletcher, A. Kisova, O. Vrana, L. Salassa, P. C. Bruijnincx, G. J. Clarkson, V. Brabec and P. J. Sadler, J. Med. Chem., 2011, 54, 3011-3026.

10 Z. Liu and P. J. Sadler, Acc. Chem. Res., 2014, 47, 1174-1185.

11 R. Payne, P. Govender, B. Therrien, C. M. Clavel, P. J. Dyson and G. S. Smith, J. Organomet. Chem., 2013, 729, 20-27.

12 A. A. Nazarov, C. G. Hartinger and P. J. Dyson, J. Organomet. Chem., 2014, 751, 251-260.

13 T. B. Hadda, M. Akkurt, M. F. Baba, M. Daoudi, B. Bennani, A. Kerbal and Z. H. Chohan, J. Enzyme Inhib. Med. Chem., 2009, 24, 457-463.

14 P. Bragg and D. Rainnie, Can. J. Microbiol., 1974, 20, 883-889.

15 R. Richards, Microbios, 1980, 31, 83-91.

16 A. Kascatan-Nebioglu, M. J. Panzner, C. A. Tessier, C. L. Cannon and W. J. Youngs, Coord. Chem. Rev., 2007, 251, 884-895.

17 B. D. Wright, P. N. Shah, L. J. McDonald, M. L. Shaeffer, P. O. Wagers, M. J. Panzner, J. Smolen, J. Tagaev, C. A. Tessier, C. L. Cannon and W. J. Youngs, Dalton Trans., 2012, 41, 6500-6506.

18 L. Oehninger, R. Rubbiani and I. Ott, Dalton Trans., 2013, 42, 3269-3284.

19 J. R. Anacona, J. Coord. Chem., 2001, 54, 355-365.

20 N. E. A. El-Gamel, J. Coord. Chem., 2009, 63, 534-543.

21 D. Scutaru, L. Tataru, I. Mazilu, M. Vata, T. Lixandru and C. Simionescu, Appl. Organomet. Chem., 1993, 7, 225-231.

22 M. Patra, G. Gasser, M. Wenzel, K. Merz, J. E. Bandow and N. Metzler-Nolte, Organometallics, 2010, 29, 4312-4319.

23 M. Wenzel, M. Patra, C. H. Senges, I. Ott, J. J. Stepanek, A. Pinto, P. Prochnow, C. Vuong, S. Langklotz, N. MetzlerNolte and J. E. Bandow, ACS Chem. Biol., 2013, 8, 1442-1450.

24 M. Patra, G. Gasser and N. Metzler-Nolte, Dalton Trans., 2012, 41, 6350-6358.

25 G. Karpin, J. S. Merola and J. O. Falkinham, Antimicrob. Agents Chemother., 2013, 57, 3434-3436.

26 L. B. Heifets, M. Iseman and P. Lindholm-Levy, Antimicrob. Agents Chemother., 1986, 30, 927-932.

27 D. M. Morris, M. McGeagh, D. De Peña and J. S. Merola, Polyhedron, 2014, 84, 120-135. 
28 U. Koelle, B. Fuss, M. Belting and E. Raabe, Organometallics, 1986, 5, 980-987.

29 M. Ehrich, personal communication.

30 J. S. Merola, D. Morris and N. De Weerd, Acta Crystallogr., Sect. E: Struct. Rep. Online, 2013, 69, m176-m176.

31 B. B. Maisuria, M. L. Actis, S. N. Hardrict, J. O. Falkinham Iii, M. F. Cole, R. L. Cihlar, S. M. Peters, R. V. Macri, E. W. Sugandhi, A. A. Williams, M. A. Poppe, A. R. Esker and R. D. Gandour, Bioorg. Med. Chem., 2011, 19, 2918-2926.
32 K. Li, L. A. Schurig-Briccio, X. Feng, A. Upadhyay, V. Pujari, B. Lechartier, F. L. Fontes, H. Yang, G. Rao, W. Zhu, A. Gulati, J. H. No, G. Cintra, S. Bogue, Y. L. Liu, K. Molohon, P. Orlean, D. A. Mitchell, L. Freitas-Junior, F. Ren, H. Sun, T. Jiang, Y. Li, R. T. Guo, S. T. Cole, R. B. Gennis, D. C. Crick and E. Oldfield, J. Med. Chem., 2014, 57, 3126-3139.

33 M. C. Babiceanu, B. A. Howard, A. C. Rumore, H. Kita and C. B. Lawrence, Front. Aquat. Microbiol., 2013, 4, 196. 